\title{
INTEGRATED QUALITY INFORMATION SYSTEM AND CHINA EXPERIENCE
}

\author{
Xiaoqing Tang, Guijiang Duan \\ School of Mechanical Engineering and Automation, Beihang University (BUAA), 37 Xueyuan \\ Road, Haidian District, Beijing 100083, China, E-mail: tangxq@buaa.edu.cn
}

\begin{abstract}
China has been becoming one of the key members in global market, and being a "plant of the world" in the past two decades. Quality is one of the essential factors for Chinese manufacturing industry to success and information technology could assist manufacturing to improve company-wide quality systems. Quality Engineering Laboratory (QEL) of Beihang University (BUAA) has been focusing on research of related methodologies, and developing technologies, and modeling for integrated quality information process to support Chinese manufacturing industry for years. An Integrated Quality Information System (IQIS) - "QQ-Enterprise" has been developed by QEL for Chinese manufacturing industries, which is a platform with generalpurpose quality functions and toolkits. A three-dimensional quality data integration model and a flexible architecture in $Q Q$-Enterprise are proposed in this paper. Success in implementing of QQ-Enterprise proved that QEL technical approach is feasible, efficient and reliable.
\end{abstract}

Key words: Quality, Integrated quality information system (IQIS), Integrated model, Flexible system architecture, Chinese manufacturing industry.

\section{INTRODUCTION}

Quality management drew a great of interests in manufacturing, service, government and education, as well as in many countries around the world. There is no exception in China manufacturing industries. China has been opening door to global market since 1970's, and market-oriented economy encouraged Chinese manufacturers adopted technologies, managing experiences and know-how. The substantial actions have been taken in improving product quality, shifting focus from production operation to product process, transiting from quality control towards total quality

Please use the following format when citing this chapter:

Tang, Xiaoqing, Duan, Guijiang, 2006, in International Federation for Information Processing (IFIP), Volume 207, Knowledge Enterprise: Intelligent Strategies In Product Design, Manufacturing, and Management, eds. K. Wang, Kovacs G., Wozny M., Fang M., (Boston: Springer), pp. 54-61. 
management (TQM), developing quality management, paying more attention in integrating ISO9000 into manufacturing process and enterprise business.

Quality data and information have been considering as an important resource to the success in quality management. Quality Engineering Laboratory (QEL) of Beihang University (original named Beijing University of Aeronautics and Astronautics) has been realized a need and focusing on the research of quality data integration in quality management since early 1990's.

To clear the concerns of Chinese manufacturing enterprises, QEL made an investigation in Shenzhen, an active manufacturing city in southern China, which included 35 questionnaires survey, covering state-owned large-size and middle-size manufacturing enterprises, joint-venture companies, and small-size or medium-size private-owned companies, and involving electronic products, electrical equipment, telecommunication, mechanical industries. The survey focused on three aspects, enterprises' attitudes and needs to QIS (Quality Information System), and current situation and practices of QIS, and problems and obstacles in implementing QIS. The Survey said: 100\% were positive answers - manufacturer needs QIS technology.

On the basis of the result of Survey, QEL started research with methodology for IQIS (Integrated Quality Information System), and modeled a system framework, and developed an Integrated Quality Information System (IQIS) - "QQ-Enterprise" for Chinese manufacturing industries.

QQ-Enterprise endues to assist manufacturing enterprises to manage their quality data in more efficient way, and to strongly support the quality system implementation in manufacturing. QEL has been encouraging Chinese manufacturers to implementing $Q Q$-Enterprise for years. $Q Q$ Enterprise is playing an important role as a quality information processing platform in improving quality system operation in the enterprises. And it is proving QEL technological approaches feasible and successful. Based on $Q Q$-Enterprise platform, more than 60 Chinese manufacturing companies have established their integrated quality information systems since the middle of 1990's. For example, a medium-size automobile accessories manufacturer in northern China had set up their IQIS in 3 months with 12 $Q Q$-Enterprise modules including QQ-QFD, QQ-CAIP, QQ-Inspection, $Q Q$-Purchase, $Q Q$-SPC, $Q Q$-Analysis, $Q Q$-Report, $Q Q$ - Improvement, $Q Q$ Assessment, QQ-Service, QQ-Document, QQ-Gauge, QQ-Audit (as in Table 1). And $Q Q$-Admin was configuring to suit the company existing quality assurance system. The tailored $Q Q$-Enterprise covered the major activities in company-wide quality assurance and management. The quality issues are 
managed in more efficient way and quality data was flowing in a more integrated way in the company.

\section{INTEGRATED QUALITY MODEL IN MANUFACTURING ENTERPRISE}

A common sense on quality management is covering the product lifecycle. It means management of quality data should be suited to companywide functions and integrated all processes of product.

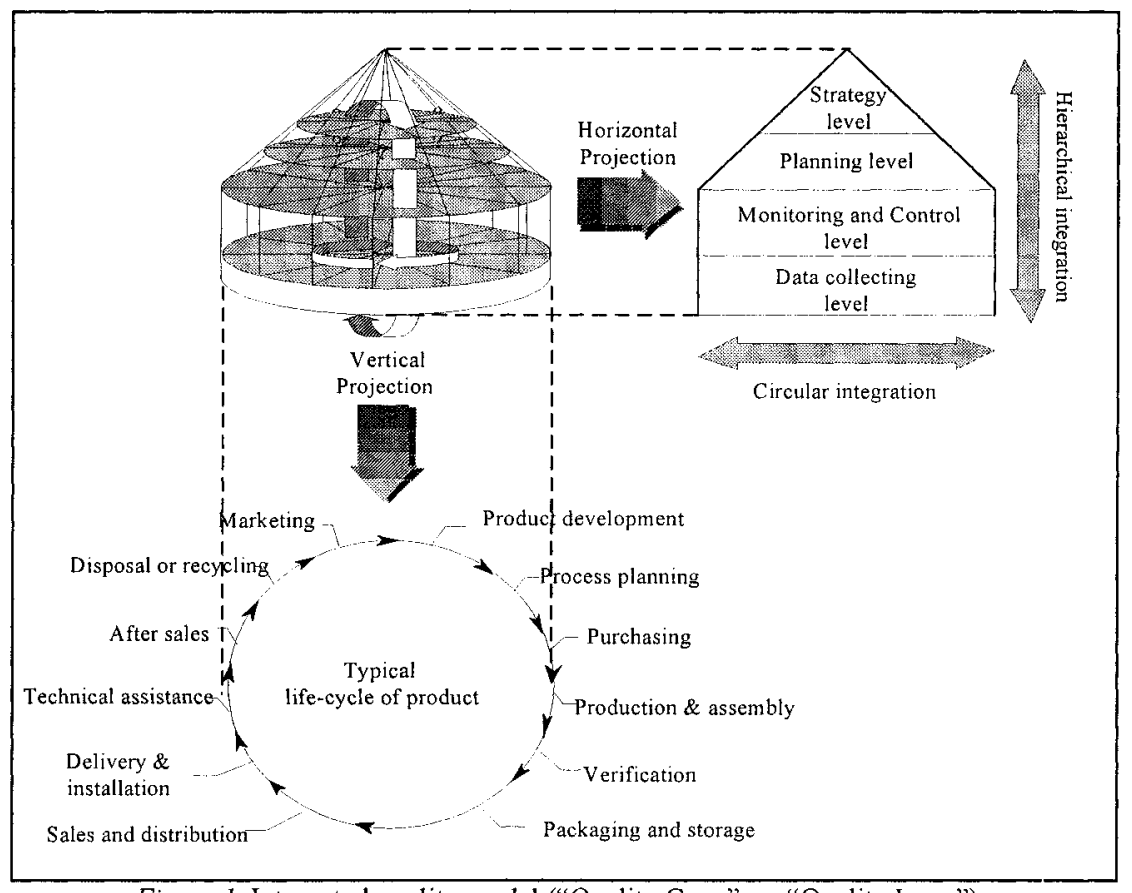

Figure l. Integrated quality model ("Quality Cone" vs. "Quality Loop").

In fighting for the fiercely competitive global market and increasingly complex manufacturing environment, efficient and accurate information processing means more to quality than ever before. Ross pointed out that accurate and timely information permits purposeful planning and decisionmaking, and drives quality and technological resources (Ross 1991). Similarly, Chang considered that implementing TQC (total quality control) philosophy in a manufacturing organization would involve the management, analysis, interface and coordination of information for all phases of the manufacturing cycle. Information integration is vital to continuous quality 
improvements in TQC environment. Integrated information system is the challenges ahead of many manufacturing organizations as they continue to improve their competitiveness (Chang, 1990). Rzeznik presented a vivid evidence to show the benefits of quality information integration. In his example, owning to timely communication and quality data sharing between shop floor operators and manufacturing engineer through Internet, inferior products are prevented from being built. Company could thus save the money and reduces production time (Rzeznik 2000).

To present quality integration, a 3-dimension model named "Quality Cone" is constructed based on the 2-dimension model "Quality Loop" in ISO9000 (Figure 1). Two individuals but cross-related integration dimensions are defined in this model. The first one is "circular integration" which covers from product life-cycle, i.e. "Quality Loop". Another integration dimension is "hierarchical integration", which is a pyramidal architecture in manufacturing organizations.

\subsection{Circular integration}

Feigenbaum indicated, "the underlying principle of the total quality view, and its basic difference from all other concepts, is that to provide genuine effectiveness, control must start with identification of customer quality requirements and end only when the product has been placed in the bands of a customer who remains satisfied" (Feigenbaum 1983). In ISO9000, the concept of quality in life cycle is formalized as a "Quality Loop" which covers all activities affecting quality. In "Quality Loop", quality data keeps flowing along with the processes and activities, and quality data would be shared by the related processes and integrated in product lifecycle.

\subsection{Hierarchical integration}

Another integration dimension is the "hierarchical integration". Corresponding to "Quality Loop", there is also a vertical integration joining the vertical layers in manufacturing enterprises. The instructions and plans are relayed up-down, as well as the quality data are gathered along the quality loop, and reported bottom-up. As moving up hierarchically, quality data is aggregated, classified, filtered and processed. The hierarchical integration will endue a vertical data flow through quality management layers, and be bridged the "gap" between different layers.

The circular integration and hierarchical integration are not isolated but interrelated. The circular integration is inter-linked by hierarchical integration. Quality data integration at both circular and hierarchical dimensions will establish a tri-dimensional quality information framework. 
"Quality Cone" model will ensure to send the right data to the right point at the right time.

\section{FLEXIBLE ARCHITECTURE OF IQIS}

As known, manufacturing enterprises are challenged by the changing global market and continuously business process reengineering (BPR). So, Integrated Quality Information System (IQIS) has to face the changing fact, and quickly reconfigured to keep pace with the dynamic environment and enterprise business process reengineering. Based on "Quality Cone" model, a flexible system infrastructure has been proposed, a general-purpose IQIS developing toolkit named $Q Q$-Enterprise has been developed.

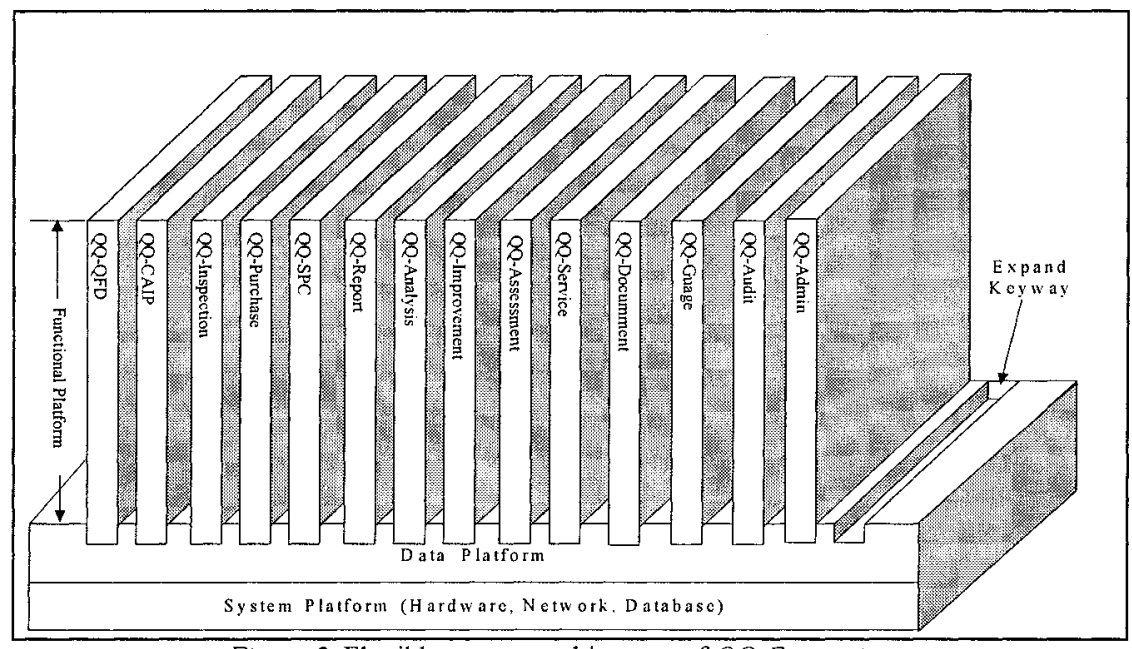

Figure 2. Flexible system architecture of $Q Q$-Enterprise.

QQ-Enterprise is constructed in a flexible architecture. As shown in Figure 2, QQ-Enterprise is organized in three levels, system platform, data platform and functional platform. System Platform embodies network and DBMS (Database Management System), serving as a supporting platform. Data Platform provides service of $Q Q$-Enterprise fundamental data, such as BOM, quality managing organization, process plans, standards and specifications, etc, which is in fact an integration platform of $Q Q$-Enterprise. Functional Platform was constructed as a slot-structure, consisting of a set of functional modules (listed in Table 1), which cover all of quality activities in product lifecycle. In advantages of modularization, these functional modules can be "assembled" together, so as to ensure high flexibility. 
Database in $Q Q$-Enterprise is able to acquire, collect, categorize, store, analyze and manipulate quality data. The quality data in $Q Q$-Enterprise is available at any time, any points, and to any person who has permit to access. $Q Q$-Enterprise is a platform for quality data sharing in internal and external enterprise environment. $Q Q$-Enterprise can communicate with $\mathrm{CAD} / \mathrm{CAM}$, ERP, CRM, SCM, etc. through reconfigurable interfaces. QQ-Enterprise can be reconfigured to suit BPR of enterprise. An open architecture with the development toolsets seems to be necessary.

Data Platform provides service to three levels - data collecting level, process monitoring and controlling level, and planning level. QQ-Enterprise Database was well organized to data logical relationship. Based on "Quality Cone" model, the quality data in different levels is effectively integrated and transferred bottom-up and top-down.

Table 1. List of functional modules in QQ-Enterprise

\begin{tabular}{|c|c|c|}
\hline Function classification & Module name & Function description \\
\hline \multirow[t]{2}{*}{ Quality plan } & $Q Q-Q F D$ & Quality function deployment \\
\hline & $Q Q-C A I P$ & Aiding to generate inspection plan \\
\hline \multirow[t]{5}{*}{ Quality control } & QQ-Inspection & $\begin{array}{l}\text { Capturing inspection data from production } \\
\text { processes }\end{array}$ \\
\hline & QQ-Purchase & $\begin{array}{l}\text { Capturing inspection data of outsourcing } \\
\text { goods }\end{array}$ \\
\hline & $Q Q-S P C$ & A series of statistical process control tools \\
\hline & $Q Q$-Analysis & $\begin{array}{l}\text { Analyzing tools to find out "quality } \\
\text { bottleneck" }\end{array}$ \\
\hline & $Q Q-$ Report & A flexible quality report generator \\
\hline Quality Improve & $Q Q$-Improvement & $\begin{array}{l}\text { A set of quality improvement tools, such as } \\
7 \text { QM tools }\end{array}$ \\
\hline Quality Assessment & $Q Q$-Assessment & An index assessment system \\
\hline \multirow[t]{4}{*}{ Quality Management } & $Q Q$-Service & $\begin{array}{l}\text { Tools for dealing with quality problems } \\
\text { after-sale }\end{array}$ \\
\hline & QQ-Document & $\begin{array}{l}\text { Archive management and search the quality } \\
\text { documents }\end{array}$ \\
\hline & QQ-Gauge & Gauging management functions \\
\hline & $Q Q-A u d i t$ & A toolkit to for quality system audit \\
\hline System Management & $Q Q-A d m i n$ & $\begin{array}{l}\text { Administrate and supervise the operation of } \\
\text { QQ-Enterprise }\end{array}$ \\
\hline
\end{tabular}




\section{IMPLEMENTING QQ-ENTERPRISE}

Implementation of $Q Q$-Enterprise is concerned with both technical and non-technical issues. QQ-Enterprise is employed to improve quality data processing but not to be able to solve all problems in enterprises. The success in implementing QQ-Enterprise requires Chinese manufacturers to adapt the existing quality system to an IQIS-featured quality system. Our experiences revealed that a thoroughly business process re-engineering (BPR) and a well-scheduled implementation guideline are effective and efficient.

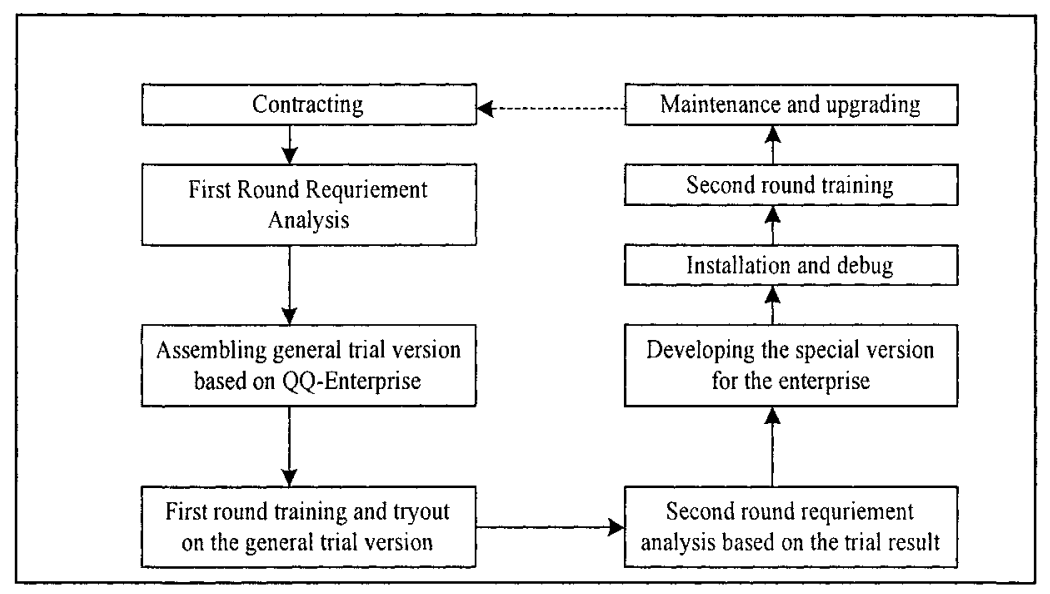

Figure 3. 1mplementing template for $Q Q$-Enterprise in Chinese manufacturer

A QQ-Enterprise implementation template, as shown in Figure 3, has been planned by QEL according to China's manufacturing circumstance, which has been proved to be feasible and useful in pilot run of $Q Q$ Enterprise in dozens of Chinese manufacturing companies. An initial system could be quickly configured according to first round requirements acquired from the user enterprise. The guideline will facilitate an effective discussion between $Q Q$-Enterprise developers and users. With the thorough discussions, the second round requirements analysis becomes more accurate and actual. By expanding the initial system a customized system will be finally constructed. This template has been proved success in practical in China. The average project cycle can be reduced to 3-6 month; meanwhile the reliability of stability of $Q Q$-Enterprise user systems would be improved.

\section{CONCLUSION}

IQIS approach can improve quality management for manufacturers. The 
survey and experience of authors reveals that, on the journey to merging into global economy for Chinese manufacturers, the needs for IQIS is increasing rapidly. Yet, their technical and managerial foundations for IQIS implementation have not been well prepared. Successfully implementing IQIS requires more efforts.

Quality data should flow in two-dimensional ways in a manufacturer, one is circular flow along with product lifecycle ("Quality Loop") and another is vertical flow across the hierarchical layers of an enterprise. A 3D quality information integration model ("Quality Cone") is aiming to endure the enterprise " $3 R$ " ability -..- "send the Right data to the Right point at the Right time".

Flexibility is important to IQIS. In global market competition, an enterprise has to improve its product and business process to suit a more dynamic environment, and improve their data accessing ability, dynamic data sharing ability and expanding ability. A flexible architecture of IQIS can support enterprises to reconfiguration and reengineering.

An integration model and flexible architecture are successfully applied in developing of $Q Q$-Enterprise, which is a general purpose IQIS system designed for Chinese manufacturers. Implementation template for $Q Q$ Enterprise practice was proved more efficient and reliable.

\section{REFERENCES}

1. Castle J A, (1998), New methodologies for integrated quality management, TQM Magazine, Vol.10, pp. 83-88.

2. Chang L C, (1990), Computer-Integrated Quality Information System, Proceedings of 1990 IEEE International Engineering Management Conference, USA, pp.85-88.

3. Duan G J and Tang X Q , (1999), Quality Information System In Dynamic Manufacturing Environment, Computer Aided Manufacturing system (CIMS), Vol.5, pp.44-48.

4. Reimann M D and Sarkis I, (1999), An architecture for integrated automated quality control, Journal of manufacturing systems, Vol.12, pp.42-48.

5. Ross, D. F., (1991), Aligning the organization for world-class manufacturing, Production and Inventory Management Journal, Second Quarter, pp.22-26.

6. Rzeznik, T, (2000), Get more from collected data, Quality Management, May 2000, pp.76-81.

7. Tang X Q , (2001), Information System supporting Enterprise Quality System, China Quality, 2001(2), pp. 35-37.

8. Tang X Q and LU Q L, (2002), Intranet/Extranet/Internet Based Quality Information Management System in Expanded Enterprise, International Journal of Advanced Manufacturing Technology, Vol.20, pp.853-858.

9. Wang X C and Tang X Q, (2001), Framework of Quality Management System for Virtual Enterprise, Manufacturing and Automation, Vol.23, pp.7-10. 